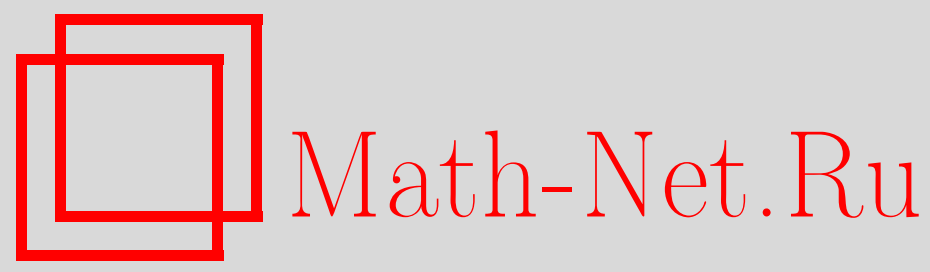

Л. Аккарди, Т. Хида, В. В. Хтай, Фоковские бозепредставления стохастических процессов, Матем. заметки, 2000, том 67, выпуск 1, 3-14

DOI: https://doi.org/10.4213/mzm808

Использование Общероссийского математического портала Math-Net.Ru подразумевает, что вы прочитали и согласны с пользовательским соглашением http://www.mathnet.ru/rus/agreement

Параметры загрузки:

IP: 18.209 .158 .208

26 апреля 2023 г., 13:54:04

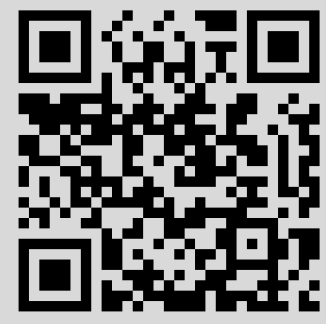




\title{
ФОКОВСКИЕ БОЗЕ-ПРЕДСТАВЛЕНИЯ СТОХАСТИЧЕСКИХ ПРОЦЕССОВ
}

\author{
Л. Аккарди, Т. Хида, В.В. Хтай
}

\begin{abstract}
Предлагается теория, классифицирующая квантовые стационарные процессы, аналогичная соответствующей теории классических стационарных процессов. Основным результатом является классификация пар классических стационарных процессов, допускающих совместное фоковское каноническое бозе-представление.

Библиографоия: 10 названий.
\end{abstract}

1. Введение. Известно, что классическая теория вероятностей и классическая теория случайных процессов могут быть вложены в квантовую теорию вероятностей [1]. Этот факт позволяет не только обобщить результаты классической теории вероятностей, но и получить новые результаты, рассматривая классические объекты с неклассической точки зрения. Некоторые публикации, появившиеся в последние годы, показьвают, что эта программа может быть осуществлена многими способами для классических диффузионных процессов в $\mathbb{R}^{n}[2]-[4]$ и на многообразиях [5], и в случае общих процессов рождения и уничтожения [4]. В настоящей заметке мы развиваем аналогичную программу для стационарных процессов. Классификация этих процессов не является полной даже в классическом случае, но в случае регулярных скалярных процессов удовлетворительная теория развита в [6] достаточно полно для того, чтобы построить нетривиальное квантовое обобщение. Обобщение, которое мы рассматриваем в настоящей заметке, мотивируется аналогией с квантовым броуновским движением (БД): известно, что с точностью до замены времени квантовые бозе-БД допускают реализацию в виде пары классических БД

$$
Q=(Q(s)), \quad P=(P(t)),
$$

которые не коммутируют, но обладают тем свойством, что их коммутатор является скалярной величиной

$$
[Q(s), P(t)]=i \min \{s, t\} .
$$

Допустим теперь, что заданы два классических стационарных процесса $X$ и $Y$, допускающие каноническое представление в смысле [6] (см. ниже уравнение (2.1)), и допустим, что $X$ можно канонически представить в виде стохастического интеграла $Q$-процесса и существует каноническоепредставление $Y$ в виде стохастического интеграла относительно $P$-процесса, где стохастические интегралы могут быть интерпретированы 
либо как классические операторозначные стохастические интегралы, либо как квантовые стохастические интегралы. Вводя обозначения, принятые в теории белого шума, мы можем записать

$$
Q(t)=\int_{0}^{t} q_{s} d s, \quad P(t)=\int_{0}^{t} p_{s} d s,
$$

где $q_{s}$ и $p_{s}$ есть белые шумы в смысле [7], удовлетворяющие коммутационным соотношениям

$$
\left[q_{s}, p_{t}\right]=i \delta(s-t)
$$

Ясно, что такое представление накладьвает некоторые ограничения на пару $X, Y$. Например, необходимьм условием для существования такого представления является то, что коммутатор любой пары случайных величин $X_{s}, Y_{t}$ является скалярным процессом специального типа (см. предложение 2). Основной результат настоящей статьи состоит в том, что это необходимое условие является также достаточньм (см. теорему 1). Более тонкой является следующая задача: насколько полно коммутатор двух процессов позволяет восстановить их каноническое представление?

С аналитической точки зрения эта задача сводится к задаче определения канонической факторизации функций в классе Харди аналитических функций. Мы доказьваем, что если два стационарных процесса являются $d$-марковскими в смысле работы [6] (см. ниже формулу (4.1)), то эта задача допускает единственное решение. Поскольку класс кратньх марковских процессов плотен в соответствующей топологии, естественно предположить, что указанньй результат справедлив также в общем случае.

Задача, которую мы изучаем, имеет естественное потенциальное применение в квантовой физике. Действительно, квантовые поля, рассматриваемые в физических моделях, являются гауссовьпи (но не марковскими) и имеют скалярные коммутаторы. Возможность представить эти поля в виде стохастических интегралов относительно квантовых БД позволяет использовать мощный аппарат исчисления белого шума как инструмент в квантовой теории поля, не прибегая к рассмотрению стохастических пределов этих полей, как это делалось до сих пор. Полное решение этой второй задачи требует более полного понимания канонических представлений многомерных (на самом деле бесконечномерньх) классических стационарных процессов. Эта проблема будет обсуждаться в последующих публикациях.

\section{2. Каноническое представление классических процессов.}

ОПРЕДЕЛЕНИЕ 1 . Пусть $X=\left\{X_{t} ; t \in[0, \infty)\right\}$ является вещественным гауссовским процессом. Предположим, что существует ядро $F(t, u)$ такое, что процесс $Y=\left\{Y_{t} ; t \in\right.$ $[0, \infty)\}$, заданньй соотношением

$$
Y_{t}=\int_{0}^{t} F(t, u) d B(u)
$$

где $B(u)$ - стандартньй броуновский процесс, удовлетворяет $B_{t}(Y)=B_{t}(B)$ для любого $t$, где $B_{t}(Y)$ и $B_{t}(B)$ являются $\sigma$-полями, порожденными $Y_{s}$ и $B(s), s \leqslant t$, соответственно, и имеет такое же конечномерное совместное распределение, как и $X$ (в этом случае, следуя терминологии, распространенной в теории вероятностей, $Y$ называется 
версией процесса $X_{t}$ ). В этом случае пара $\{F(t, u), u \leqslant t ; B(t)\}$ (обозначаемая через $\{F(t, u), B\})$ назьвается каноническим представлением $X$, а $F(t, u)$ является каноническим ядром.

В существующей литературе термин каноническое представление иногда используется для более сильного условия, состоящего в том, что $X_{t}$ сам представляется в виде $(2.1)$, т.е. $Y_{t}=X_{t}$. В этом случае введенное вьше броуновское движение $B$ является процессом обновления для $X$. Если процесс $X_{t}$ стационарен, можно выбрать $F(t, u)=F(t-u)$.

ОПРЕДЕЛЕНИЕ 2. Будем говорить, что пара классических стационарных процессов $\widetilde{X}, \widetilde{Y}$ допускает совместное представление, если их каноническое представление может быть построено в одном и том же вероятностном пространстве.

Другими словами, $\widetilde{X}, \widetilde{Y}$ допускает совместноепредставление, если в указанном вьше смысле существуют версии $X, Y$ процессов $\widetilde{X}, \widetilde{Y}$ соответственно такие, что канонические представления выражаются в виде

$$
X_{t}=\int G(t-u) W(u) d u, \quad Y_{t}=\int F(t-u) W(u) d u
$$

с помощью одного и того же белого шума $\{W(u)\}$. Ядра $G(u)$ и $F(u)$ обращаются в нуль при $u<0$.

Заметим, что для скалярного процесса, допускающего каноническое представление, всегда может быть построено совместное представление, поэтому определение 2 является нетривиальным только в случае многомерных процессов.

В настоящей заметке мы широко используем терминологию квантовой теории вероятностей. Понятие стохастического процесса используется в том же смысле, как и в [1]. Квантовьй бельй шум (1.4) рассматривается в фоковском пространстве над $L^{2}(\mathbb{R})$ в соответствии с тем фактом, что мы рассматриваем только квантовые аналоги скалярных процессов, допускающих операторную реализацию в этом фоковском пространстве. В частности, под классическим прочессом мы понимаем семейство $\left(X_{t}\right)(t \in \mathbb{R})$ самосопряженных операторов на введенном выше фоковском пространстве таких, что экспоненты $\exp \sum_{j=1}^{n} a_{j} X_{t_{j}}$ корректно определены для любого конечного семейства вещественных чисел $t_{1}, \ldots, t_{n}, a_{1}, \ldots, a_{n}$, и семейство таких экспонент порождает абелеву алгебру фон Неймана. Термин стационарный для таких процессов всегда относится к их распределениям относительно вакуумного вектора $\Phi$, так что условие стационарности означает, что

$$
\left\langle\Phi, \exp \sum_{j=1}^{n} a_{j} X_{t_{j}+s} \Phi\right\rangle=\left\langle\Phi, \exp \sum_{j=1}^{n} a_{j} X_{t_{j}} \Phi\right\rangle
$$

для любого финитного семейства вещественных чисел $t_{1}, \ldots, t_{n}, a_{1}, \ldots, a_{n}$ и любого вешественного числа $s$.

ОПРЕДЕЛЕНИЕ 3. Пара классических стационарных процессов $X, Y$ допускает совместное фоковское представление, если существует фоковский бельй бозе-шум

$$
\Gamma\left(\mathcal{H}_{1}\right)=:\left\{\mathcal{H}, \Phi, q_{t}, p_{t}\right\}
$$


такой, что $X$ и $Y$ могут быть представлены (с точностью до изоморфизма конечно-мерных распределений) в виде

$$
X_{t}=\int G(t-u) q_{u} d u, \quad Y_{t}=\int F(t-u) p_{u} d u
$$

для двух вещественных функций $F$ и $G$. Представление (2.4) называется каноническим, если представление $X$ является каноническим для белого шума $q$ и представление $Y$ является каноническим для белого шума $p$. Заметим, что поскольку процессы являются классическими, имеет смысл говорить о их канонических представлениях в смысле определения 1.

ПРЕДЛОЖЕНИЕ 1. Два классических стационарных прочесса $X, Y$ допускают совместное фоковское представление тогда и только тогда, когда они допускают совместное представление в классическом смысле.

ДокАЗАТЕЛЬСтво. Напомним, что мы отождествляем классический бельй шум с $p$-процессом в фоковском пространстве, и пусть $\Gamma(i)$ обозначает преобразование $\Phi$ рье-Гаусса. Тогда

$$
q_{u}=\Gamma(i) p_{u} \Gamma(i)^{*}, \quad u \in \mathbb{R} .
$$

Процесс $\tilde{Y}_{t}=\Gamma(i) Y_{t} \Gamma(i)^{*}$ имеет такое же вакуумное распределение, как и $Y_{t}$, поскольку $\Gamma(i) \Phi=\Phi$. Если $Y_{t}$ имеет вид $(2.4)$, тогда $\widetilde{Y}_{t}$ имеет вид

$$
\tilde{Y}_{t}=\int F(t-u) q_{u} d u
$$

Поскольку унитарньй изоморфизм отображает условное ожидание процесса $Y$ в соответствующее ожидание процесса $\widetilde{Y}$, отсюда следует, что если представление $Y$ каноническое, то (2.5) изоморфно каноническому представлению $\widetilde{Y}$. Из этого замечания немедленно следует доказьваемое утверждение, поскольку оба процесса $X$ и $\widetilde{Y}$ реализованы в одной и той же абелевой алгебре фон Неймана, т.е. в одном и том же вероятностном пространстве с одной и той же фильтрацией.

Следующий факт является элементарньм и хорошо известным.

ЛЕмма 1. Пространство интегрируемых функиий, обращающихся в нульналевой полупрямой, является алгеброй относительно свертки.

ДоКАЗАТЕЛЬСтвО. Пусть $F(t)$ и $G(t)$ - интегрируемые функции при $t<0$. Тогда их свертки корректно определены и интегрируемы [8]. Рассмотрев тождество

$$
F * G(t)=\int_{-\infty}^{t} F(t-s) G(s) d s,
$$

мы видим, что $F * G$ обращается в нуль на левой полупрямой.

ЗАмЕчАниЕ 1. Следствием предыдущей леммы является то, что пространство преобразований Фурье интегрируемых функций, обращающихся в нуль на левой полупрямой, является алгеброй относительно поточечного умножения. В дальнейшем изложении мы всегда используем обозначение $\widehat{F}$ для преобразования Фурье функции $F$. 
ПРЕДЛОЖЕНИЕ 2. Если два квантовых стационарных процесса $X$ и $Y$ допускают совместное фоковское бозе-представление, то в этом представлении для всех вещественных чисел $s, t$ области определения $X_{s}$ и $Y_{t}$ имеют плотное пересечение и их коммутатор имеет вид

$$
\left[X_{s}, Y_{t}\right]=i \Theta(s-t)=i \int e^{-i(s-t) \lambda} \widehat{\Theta}(\lambda) d \lambda, \quad s, t \in \mathbb{R}
$$

где $\widehat{\Theta}$ - Фурье преобразование функиии, которая обращается в нуль на левой полупрямой, а коммутатор понимается в слабом смысле на пересечении областей определения. Более того, функиия $\Theta$ однозначно определена тождеством

$$
\widehat{\Theta}(\lambda)=\widehat{G}(\lambda) \overline{\widehat{F}(\lambda)}
$$

где F и $G$ определены формулой (2.2).

ДокАЗАТЕЛЬСтво. Пусть представления $X$ и $Y$ имеют вид (2.4). Тогда, вводя спектральное представление процессов белого шумов $q$ и $p$

$$
q_{\lambda}=\frac{1}{\sqrt{2 \pi}} \int e^{i t \lambda} q_{t} d t, \quad p_{\lambda}=\frac{1}{\sqrt{2 \pi}} \int e^{-i t \lambda} p_{t} d t
$$

мы можем переписать коммутаторы в виде

$$
\left[X_{s}, Y_{t}\right]=i \int_{-\infty}^{t \wedge s} G(s-u) F(t-u) d u=i \int_{-\infty}^{\infty} e^{-i(s-t) \lambda} \widehat{G}(\lambda) \overline{\widehat{F}(\lambda)} d \lambda
$$

поскольку $F$ и $G$ являются вещественньми. Функции $\widehat{\Theta}(\lambda):=\widehat{G}(\lambda) \overline{\widehat{F}(\lambda)}$ являются преобразованиями $\Phi$ урье $(G * F)(t)$, обращающимися в нуль на левой полупрямой.

ОПРЕДЕЛЕНИЕ 4. Совместное гильбертово представление пары классических стационарных процессов $X, Y$ есть пара $\{\mathcal{H}, \Phi\}$, где $\mathcal{H}$ - гильбертово пространство и где $\Phi$ - единичный вектор в $\mathcal{H}$ такой, что для любого $t \in \mathbb{R} \quad X_{t}$ и $Y_{t}$ имеют представление как операторы в $\mathcal{H}$ (также обозначаемьми через $X_{t}, Y_{t}$ для простоты).

ОПредЕЛЕниЕ 5. Два гильбертовых представления $\{\mathcal{H}, \Phi\}$ и $\left\{\mathcal{H}^{\prime}, \Phi^{\prime}\right\}$ называются әквивалентными, если существует унитарньй изоморфизм $U: \mathcal{H} \rightarrow \mathcal{H}^{\prime}$ такой, что $U \Phi=\Phi^{\prime}$ и что $U$ представляет действие $X$ и $Y$ в $\mathcal{H}$ и $\mathcal{H}^{\prime}$ соответственно.

ЗАмЕчАнИЕ 2. Из определения 5 следует, что любая пара стохастических процессов имеет совместное гильбертово представление. Для этой цели достаточно рассмотреть стандартное гильбертово представление $\left\{\mathcal{H}_{X}, \Phi_{X}\right\},\left\{\mathcal{H}_{Y}, \Phi_{Y}\right\}$ процессов $X$ и $Y$, определенных в лемме 1 , и построить их тензорное произведение

$$
\mathcal{H}:=\mathcal{H}_{X} \otimes \mathcal{H}_{Y} ; \quad \Phi:=\Phi_{X} \otimes \Phi_{Y}
$$

С другой стороны ясно, что если процессы классические, это представление в общем случае не является каноническим. 
Наша цель состоит в решении следующей задачи.

Пусть задано совместное гильбертово представление $\{\mathcal{H}, \Phi\}$ пары классических стационарных процессов $X$ и $Y$. При каких условиях это представление эквивалентно фоковскому бозе-представлению $X$ и $Y$ ?

Предложение 1 и предложение 2 дают для этого два необходимых условия. Именно,

i) $X, Y$ должны иметь канонические представления;

ii) коммутатор $X$ и $Y$ должен быть корректно определен в слабом смысле на плотной области, и на этой области определения он должен иметь вид (2.6), (2.9).

3. Достаточность условий. Пусть $X=\left(X_{t}\right), Y=\left(Y_{t}\right)$ являются стационарными классическими стохастическими процессами, реализованными как операторы в гильбертовом пространстве $\mathcal{H}$ с единичным вектором $\Phi$ таким, что

$$
\begin{aligned}
\left\langle\Phi, f\left(\left\{X_{t}\right\}\right) \Phi\right\rangle & =E\left(f\left(\left\{X_{t}\right\}\right)\right), \\
\left\langle\Phi, f\left(\left\{Y_{t}\right\}\right) \Phi\right\rangle & =E\left(f\left(\left\{Y_{t}\right\}\right)\right),
\end{aligned}
$$

где $f\left(\left\{X_{t}\right\}\right)$ обозначает произвольньй функционал процесса $X_{t}$ и аналогично для $Y_{t}$. Заметим, что левая часть (3.1), (3.2) корректно определена спектральной теоремой для любой борелевской функции $f$ на $\mathbb{R}$, зависящей только от конечного числа случайных переменных. Такое определение может быть распространено на произвольные измеримые функционалы с помощью канонического определения абелевой алгебры, порожденной случайными переменными с алгеброй ограниченных функционалов классического процесса.

Предположим, кроме того, что $X$ и $Y$ имеют совместное представление $\{\mathcal{H}, \Phi\}$ в смысле определения 4:

$$
\begin{aligned}
X_{t} & =\int e^{-i t \lambda} \widehat{G}(\lambda) q_{\lambda} d \lambda \\
Y_{t} & =\int e^{i t \lambda} \overline{\widehat{F}(\lambda)} p_{\lambda} d \lambda
\end{aligned}
$$

где $q_{\lambda}$ и $p_{\lambda}-$ стандартные белые шумы относительно вектора $\Phi$ и, кроме того, каждое из представлений (3.3) изоморфно каноническому представлению соответствующего классического процесса. Предположим дополнительно, что для процессов

$$
\frac{1}{\sqrt{2}}\left(q_{\lambda}+i p_{\lambda}\right)=a_{\lambda}, \quad \frac{1}{\sqrt{2}}\left(q_{\lambda}-i p_{\lambda}\right)=a_{\lambda}^{+}
$$

вакумньй вектор $\Phi$ принадлежит области определения алгебры полиномов, порожденных $a_{\lambda}^{ \pm}$(в смысле распределений), и

$$
a_{\lambda} \Phi=0
$$

Заметим, что мы не делаем никаких предположений о коммутационных соотношениях между двумя белыми шумами $p$ и $q$. Наша цель - вывести эти коммутационные соотношения из коммутационных соотношений для процессов $X$ и $Y$. Таким образом будет доказано, что необходимые условия, приведенные вьше в предыдущем параграфе, являются также достаточньми. 
Лемма 2. Пусть $M, N$ - операторозначные меры на $\mathbb{R} \times \mathbb{R}$ такие, что для любых $и \in \mathbb{R}^{2}$ выполнено

$$
\int_{\mathbb{R}^{2}} e^{i u \cdot \alpha} M(d \alpha)=\int_{\mathbb{R}^{2}} e^{i u \cdot \alpha} N(d \alpha),
$$

где $u \cdot \alpha$ - скалярное произведение в $\mathbb{R}^{2}$. Тогда $M=N$.

ДокАЗАТЕльство. Утверждение следует из рассмотрения матричных элементов тождества (3.6) и использования свойства единственности преобразования Фурье комплексных мер.

Пусть теперь предполагается, что условие (2.6) вьполнено для некоторой функции $\Theta$, удовлетворяющей условиям предложения 2. Тогда явное вычисление коммутатора $X$ и $Y$ с помощюю уравнения (3.3) дает:

$$
\begin{aligned}
{\left[X_{s}, Y_{t}\right] } & =\iint e^{-i s \lambda} e^{i t \lambda^{\prime}} \widehat{G}(\lambda) \overline{\widehat{F}\left(\lambda^{\prime}\right)}\left[q_{\lambda}, p_{\lambda^{\prime}}\right] d \lambda d \lambda^{\prime} \\
& =i \int e^{-i(s-t) \lambda} \widehat{\Theta}(\lambda) d \lambda=i \iint e^{-i s \lambda} e^{i t \lambda^{\prime}} \widehat{\Theta}(\lambda) \delta\left(\lambda-\lambda^{\prime}\right) d \lambda d \lambda^{\prime}
\end{aligned}
$$

из леммы 2 следует, что

$$
\widehat{G}(\lambda) \overline{\widehat{F}\left(\lambda^{\prime}\right)}\left[q_{\lambda}, p_{\lambda^{\prime}}\right]=i \widehat{\Theta}(\lambda) \delta\left(\lambda-\lambda^{\prime}\right)
$$

в смысле распределений. Если произведение $\widehat{G}(\lambda) \overline{\widehat{F}\left(\lambda^{\prime}\right)}$ в тождестве $(3.8)$ равно нулю на множестве нулевой меры Лебега, то мы можем изменить это значение, не изменяя (3.8). Если произведение равно нулю на множестве положительной меры Лебега на диагонали $\lambda=\lambda^{\prime}$, то также $\widehat{\Theta}(\lambda)$ должно обращаться в нуль. Таким образом, принимая в качестве определения, что следующее отношение равно нулю в тех точках диагонали, где обращается в нуль знаменатель, мы можем написать:

$$
\left[q_{\lambda}, p_{\lambda^{\prime}}\right]=i \frac{\widehat{\Theta}(\lambda)}{\widehat{G}(\lambda) \overline{\widehat{F}\left(\lambda^{\prime}\right)}} \delta\left(\lambda-\lambda^{\prime}\right) .
$$

Теперь, используя белые шумы $q_{\lambda}, p_{\lambda^{\prime}}$ в $(3.3)$, определим процессы $q_{t}$ и $p_{t}$ с помощюю соотношения (2.8). Тогда имеет место

$$
\left[q_{\lambda}, p_{\lambda^{\prime}}\right]=\frac{1}{2 \pi} \iint e^{i s \lambda} e^{-i t \lambda^{\prime}}\left[q_{s}, p_{t}\right] d s d t .
$$

Поэтому, вычисляя обратное преобразование Фурье (3.9), мы находим:

$$
\begin{aligned}
{\left[q_{s}, p_{t}\right] } & =\frac{1}{2 \pi} i \iint e^{-i s \lambda} e^{i t \lambda^{\prime}} \frac{\widehat{\Theta}(\lambda)}{\widehat{G}(\lambda) \widehat{\widehat{F}\left(\lambda^{\prime}\right)}} \delta\left(\lambda-\lambda^{\prime}\right) d \lambda d \lambda^{\prime} \\
& =\frac{1}{2 \pi} i \int e^{-i(s-t) \lambda} \frac{\widehat{\Theta}(\lambda)}{\widehat{G}(\lambda) \widehat{\widehat{F}(\lambda)}} d \lambda .
\end{aligned}
$$

Отсюда следует, что

$$
\frac{1}{2 \pi} \int e^{-i(s-t) \lambda} \frac{\widehat{\Theta}(\lambda)}{\widehat{G}(\lambda) \overline{\widehat{F}(\lambda)}} d \lambda=g(s-t)
$$

для некоторой функции или распределения $g(s-t)$. Мы хотим доказать, что $g(s-t)=$ $\delta(s-t)$. 
Лемма 3. Пусть заданы гильбертово пространство $\mathcal{H}$ и единичный вектор $\Phi$, и пусть заданы два самосопряженных оператора на $\mathcal{H}$ и $q, p$, имеющие стандартнье Гауссовы Ф-распределения (см. определение 4). Предположим дополнительно, что $[q, p]=i c, c \neq 0, c \in \mathbb{R}, u$ что эти соотночения мохнно әкспоненцировать в вейлевские соотношения

$$
e^{i s p} e^{i t q}=e^{-i s t} e^{i t q} e^{i s p}
$$

Тогда $c=1$.

ДокАЗАТЕЛЬСТво. Предположим противное. Возможно, заменяя $p$ на $q$, мы можем считать, что $c>0$. Тогда новая пара

$$
P:=\frac{p}{\sqrt{c}}, \quad Q:=\frac{q}{\sqrt{c}}
$$

удовлетворяет $[Q, P]=i$. В силу предположения о возможности экспоненцирования коммутационных соотношений между $q$ и $p$ отсюда следует, что с точностью до кратности представления система $\{\mathcal{H}, \Phi, Q, P\}$ изоморфна шрёдингеровскому представлению. Отсюда следует, что $Q, P$ являются $\Phi$-стандартньпи гауссовыми. Поэтому

$$
\left\langle\Phi, e^{i q \lambda} \Phi\right\rangle=\left\langle\Phi, e^{i \sqrt{c} \lambda Q} \Phi\right\rangle=\int e^{i \sqrt{c} x \lambda} \frac{e^{-\frac{x^{2}}{2}}}{(2 \pi)^{\frac{1}{2}}} d x=\int e^{i y \lambda} \frac{e^{-\frac{y^{2}}{2 c}}}{(2 \pi c)^{\frac{1}{2}}} d y
$$

Следовательно, $q$ является стандартной $\Phi$-гауссовой случайной величиной с нулевым средним и дисперсией $c$. Но в соответствии с предположением $q$ является стандартной гауссовой величиной с $c=1$. Рассмотрим этот результат в приведенных выше обозначениях в случае, когда

$$
\left[q_{s}, p_{t}\right]=i g(s-t)
$$

Вводя тестовые функции $\varphi, \psi$ и связанные с ними сглаженные шумы, имеем

$$
q(\varphi)=\int \varphi(s) q_{s} d s, \quad p(\psi)=\int \psi(s) p_{s} d s
$$

(3.10) эквивалентно $[q(\varphi), p(\psi)]=i \iint \varphi(s) g(s-t) \psi(t) d s d t$.

ТЕОрема 1. Пусть $X$ и $Y$ определены формулами (3.3) с $q_{\lambda}$ и $p_{\lambda}$, удовлетворяющими (3.5), и условиями, определенными после формуль (3.3). Если существует скалярная интегрируемая функция $\widehat{\Theta}$ такая, что коммутатор $X$ и $Y$ имеет вид

$$
\left[X_{s}, Y_{t}\right]=i \int e^{-i(s-t) \lambda} \widehat{\Theta}(\lambda) d \lambda
$$

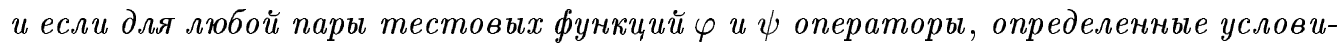
ями (3.10), удовлетворяют условиям леммы 3 , то белье шумы $q_{\lambda}, p_{\lambda}$ являются спектральными представлениями фоковского белого бозе-иума $q_{t}, p_{t}$. 
ДокАЗАТЕльство. Тождество поляризации однозначно определяет коммутатор $[q(\varphi), p(\psi)]$ в случае $\varphi=\psi$. Зафиксируем $\varphi$ и предположим, что

$$
\int \varphi^{2}(s) d s=1
$$

Тогда согласно лемме $3 q(\varphi), p(\varphi)$ являются стандартными $\Phi$-гауссовыми и

$$
[q(\varphi), p(\psi)]=i \iint \varphi(s) g(s-t) \psi(t) d s d t .
$$

С помощью леммы 3 мы доказываем, что из (3.12) следует, что

$$
\iint \varphi(s) g(s-t) \varphi(t) d s d t=1
$$

С помощью тождеств поляризации можно доказать, что условия (3.12) и (3.13) эквивалентны условию

$$
\iint \varphi(s) g(s-t) \psi(t) d s d t=\iint \varphi(s) \delta(s-t) \psi(t) d s d t
$$

для любых $\varphi$ и $\psi$. Отсюда следует

$$
g(s-t)=\delta(s-t)
$$

Но согласно нашему предположению и (2.8) процессы (3.4) уничтожают вакуум. В сочетании с коммутационными соотношениями (3.10) этого достаточно, чтобы гарантировать, что пара $q_{t}, p_{t}$ изоморфна стандартному фоковскому белому бозе-шуму.

4. Каноническое свойство и кратное марковское свойство. Перед тем как перейти к следующей задаче, мы приведем краткий обзор канонических представлений классических гауссовских процессов.

Предположим, что процесс $X_{t}$ задан соотношением (2.2). В этом случае известно, что преобразование Фурье $\widehat{G}(\lambda)$ функции $G$ принадлежит классу Харди (см. [9]) и, следовательно, является краевым значением функции $\widehat{G}(w)$, аналитической в нижней полуплоскости $C_{-}$.

ТЕОРемА 2. Пусть $X, Y$ являются классическими стохастическими прочесса$\mathcal{м и , ~}\{\mathcal{H}, \Phi\}$ - гильбертово пространство представления пары $X, Y$ и предположим, что условия теоремы 1 выполнены. Тогда, обозначая

$$
\widehat{\Theta}=C_{\Theta} B_{\Theta} S_{\Theta}, \quad \widehat{G}=C_{1} B_{1} S_{1}, \quad \widehat{F}=C_{2} B_{2} S_{2}
$$

канонические декомпозичии $\widehat{\Theta}, \widehat{G}$ и $\widehat{F}$ соответственно, имеем, что пара $\{\mathcal{H}, \Phi\}$ изоморфна в смысле определения 5 фоковскому бозе-представлению $\{X, Y\}$, если и только если

$$
C_{\Theta}=C_{1} \bar{C}_{2} ; \quad B_{\Theta}=B_{1} \bar{B}_{2} ; \quad S_{\Theta}=S_{1} \bar{S}_{2}
$$


ДокАЗАТЕЛЬСТВО. Утверждение следует из единственности декомпозиции функций из класса Харди.

Теперь мы предположим, что $X_{t}$ является кратным марковским процессом порядка $N<+\infty$. Представляется весьма естественньп предположение о том, что $X_{t}$ является математической моделью некоторых физических явлений. Для определения кратного марковского свойства (классического) гауссова процесса мы отсылаем читателя к [6, глава 5$]$.

Это предположение эквивалентно следуюшему факту: функция $\widehat{G}(\lambda)$ представляется как рациональная функция вида

$$
C(\lambda)=\frac{Q(i \lambda)}{P(i \lambda)}, \quad-\infty<\lambda<\infty,
$$

где $P$ и $Q$ - полиномы степени $Q<$ степени $P=N$, которые не имеют корней с положительными вещественными частями (см. $[6, \S 5.4])$.

5. Факторизация. Перед тем как рассмотреть факторизацию функции $\widehat{\Theta}$, мы обобщим определение классического стохастического кратного марковского процесса на некоммутирующие процессы.

Пусть $X_{t}$ и $Y_{t}-$ классические стохастические процессы:

$$
X_{t}=\int^{t} G(t, u) q_{u} d u, \quad Y_{t}=\int^{t} F(t, u) p_{u} d u
$$

где $G$ и $F-$ канонические ядра.

ОПРЕДЕЛЕНИЕ 6. Квантовьй стохастический процесс, заданный парой $X$ и $Y$, называется кратным марковским проиессом, если вакуумные распределения $X_{t}$ и $Y_{t}$ соответственно определяют кратные марковскиегауссовы процессы в классическом смысле.

Переходя к стационарным квантовым стохастическим процессам $X_{t}$ и $Y_{t}$, заданным (2.4), мы предполагаем, что они являются кратными марковскими процессами. Тогда согласно $[6$, гл. 5] канонические ядра $G$ и $F$ являются ядрами типа Гурса. Это означает, что их преобразования Фурье выражаются через рациональные функции вида (4.1), например,

$$
\widehat{G}(\lambda)=\frac{Q_{1}(i \lambda)}{P_{1}(i \lambda)}, \quad \widehat{F}(\lambda)=\frac{Q_{2}(i \lambda)}{P_{2}(i \lambda)},
$$

где $P_{i}, Q_{i}, i=1,2$, являются полиномами. Дополнительно мы предполагаем, что эти полиномы не имеют нулей на вешественной прямой.

Теперь мы можем перейти к задаче факторизации функции $\widehat{\Theta}(\lambda)$.

Согласно предположению $\widehat{\Theta}(\lambda)$ имеет такой же вид, как и в $(2.7)$. Заметим, что несимметричность $X$ и $Y$ в формуле (2.7) играет в определении 4 роль спектрального преобразования квантового белого шума, которьй был введен в связи с необходимостью сохранения коммутационных соотношений.

Следовательно, мы должны получить

$$
\widehat{\Theta}(\lambda)=\frac{Q_{1}(i \lambda) \overline{Q_{2}(i \lambda)}}{P_{1}(i \lambda) \overline{P_{2}(i \lambda)}} .
$$


Теперь наша задача состоит в том, чтобы определить $P_{i}$ и $Q_{i}, i=1,2$. Заданная функция $\widehat{\Theta}$ должна быть произведением двух функций $\widehat{\Theta}_{1}(\lambda)$ и $\widehat{\Theta}_{2}(\lambda)$, являющихся краевьми значениями $\widehat{\Theta}_{1}(w), \widehat{\Theta}_{2}(w)$, которые в свою очередь являются функциями $w$ и $\bar{w}$ соответственно. Рассмотрим полюсы $\widehat{\Theta}$ в $C^{+}$. Тогда они должны быть корнями $P_{1}$. Нули $\widehat{\Theta}$ в $C^{+}$должны быть нулями $Q_{1}$. Аналогично, полюсы и нули в $C^{-}$должны быть нулями $\bar{P}_{2}$ и $\bar{Q}_{2}$ соответственно.

Следовательно, $P_{i}, Q_{i}, i=1,2$, определены с точностью до постоянных. Это означает, что $\widehat{G}$ и $\widehat{F}$ определены с точностью до постоянных.

Как было замечено, кратности полиномов $P_{1}$ и $P_{2}$ равны кратностям марковских процессов $X_{t}$ и $Y_{t}$ соответственно.

ТЕОремА 3. Предположим, что пара квантовых стохастических прочессов $X_{t}$ $u Y_{t}$ являются кратными марковскими прочессами и удовлетворяют условиям теоремы 1. Тогда если задан коммутатор $X_{t} u Y_{t}$, то кратности марковских процессов $X_{t}$ и $Y_{t}$ определень однозначно каноническими представлениями $X_{t} u$ $Y_{t}$ вида (2.4) с точностью до постоянного множителя.

6. Заключение. Нам хотелось бы завершить статью одним весьма предварительным комментарием к случаю, когда возникают неканонические представления.

В этом случае существует много способов факторизации функции $\widehat{\Theta}(\lambda)$, определяющей коммутатор двух процессов. Например, если мы предполагаем, что $X, Y$ являются кратньми гауссовыми процессами и, кроме того, что не существует сингулярных множителей в $\widehat{\Theta}, \widehat{G}$ и $\widehat{F}$, то мы можем написать $\widehat{G}(\lambda)=C_{1}(\lambda) B_{1}(\lambda), \widehat{F}(\lambda)=C_{2}(\lambda) B_{2}(\lambda)$, где $C_{i}$ являются рациональными функциями, соответствующими каноническому ядру, и $B_{i}$ является произведением Бляшке, $i=1,2$. В этом случае $\widehat{\Theta}(\lambda)=C_{1}(\lambda) \overline{C_{2}(\lambda)}$. $B_{1}(\lambda) \overline{B_{2}(\lambda)}$ и известно, что

$C_{1}(\lambda)$ не имеет нулей и полюсов нижней полуплоскости $C^{-}$;

$\overline{C_{2}(\lambda)}$ не имеет нулей и полюсов в верхней полуплоскости $C^{+}$;

$B_{1}(\lambda)$ имеет нули в $C^{-}$и полюсы в $C^{+}$;

$\overline{B_{2}(\lambda)}$ имеет нули в $C^{+}$и полюсы в $C^{-}$.

Если мы рассмотрим полюсы $\widehat{\Theta}$ в $C^{+}$таким же образом, как в каноническом случае, то эти полюсы могут быть полюсами типа $C_{1}$ или $B_{1}$. Аналогичная неоднозначность может наблюдается для нулей и полюсов в $C^{-}$.

Нам хотелось бы высказать предположение о том, что при некоторых условиях оптимальности на $|\widehat{\Theta}(w)|$ в том же смысле, как в [10], возможно индивидуализировать вклад сомножителей Бляшке в нули и полюсы. Эта проблема будет рассмотрена нами позже.

Авторы выражают признательность рецензенту за внимательное прочтение статьи и подробные замечания, позволившие сделать работу более понятной.

\section{СПИСОК ЦИТИРОВАННОЙ ЛИТЕРАТУРЫ}

[1] Accardi L., Fagnola F. Quaegebeur quantum stochastic calculus // J. Funct. Anal. 1992. V. 104. P. 149-197; // Volterra preprint. 1990. № 18.

[2] Bhat B. V.R., Fagnola F., Sinha B. On quantum extensions of semigroups of Brownian motion on a half-line // Russian J. of Math. Phys. 1996. V. 4. № 3. P. 13-28.

[3] Chebotarev A. M. Minimal solution in classical and quantum stochastics // Quantum Probability and Related Topics. V. 7. Singapore: World Scientific, 1992. P. 79-97. 
[4] Fagnola F., Monte R. On quantum extension of the semigroup of Bessel process quantum extensions of semigroups // Math. Notes. 1996. V. 60. № 4. P. 519-537.

[5] Accardi L., Mohari A. On the structure of classical and quantum flows // Journ. Funct. Anal. 1996; // Volterra Preprint. 1994. № 167.

[6] Hida T., Hitsuda M. Gaussian Processes // Amer. Math. Soc., Providence, R. I. 1993.

[7] Hida T. Brownian Motion. New York: Springer-Verlag, 1980.

[8] Kolmogorov A. N., Fomin S. V. Introductory Real Analysis. New York: Dover Publications, 1970.

[9] Hoffman K. Banach Spaces of Analytic Functions. Prentice-Hall, 1962.

[10] Htay W. W. Optimalities for random functions: Lee-Wiener's network and non-canonical representation of stationary Gaussian processes // Nagoya Math. J. 1998. V. 149. P. 9-17.

Университет Тор Вергата, Рим,

Поступило

Университет г. Мейжо,

23.08.1999

E-mail: accardi@volterra.mat.utovrm.it, thida@meijo-u.ac.jp 